\title{
Persistent 7-tesla phase rim predicts poor outcome in new multiple sclerosis patient lesions
}

\author{
Martina Absinta, ${ }^{1,2}$ Pascal Sati, ${ }^{1}$ Matthew Schindler, ${ }^{1}$ Emily C. Leibovitch, ${ }^{1}$ Joan Ohayon, ${ }^{1}$ Tianxia Wu, ${ }^{1}$ Alessandro Meani, ${ }^{2}$ \\ Massimo Filippi, ${ }^{2}$ Steven Jacobson, ${ }^{1}$ Irene C.M. Cortese, ${ }^{1}$ and Daniel S. Reich ${ }^{1}$
}

'Division of Neuroimmunology and Neurovirology, National Institute of Neurological Disorders and Stroke (NINDS), NIH, Bethesda, Maryland, USA. ${ }^{2}$ Neuroimaging Research Unit, Institute of Experimental Neurology, Division of Neuroscience, San Raffaele Scientific Institute, Vita-Salute San Raffaele University, Milan, Italy.

BACKCROUND. In some active multiple sclerosis (MS) lesions, a strong immune reaction at the lesion edge may contain growth and thereby isolate the lesion from the surrounding parenchyma. Our previous studies suggest that this process involves opening of the blood-brain barrier in capillaries at the lesion edge, seen on MRI as centripetal contrast enhancement and a colocalized phase rim. We hypothesized that using these features to characterize early lesion evolution will allow in vivo tracking of tissue degeneration and/or repair, thus improving the evaluation of potential therapies for chronic active lesions.

METHODS. Centripetally and centrifugally enhancing lesions were studied in 17 patients with MS using 7-tesla MRI. Highresolution, susceptibility-weighted, T1-weighted (before/after gadolinium), and dynamic contrast-enhanced scans were acquired at baseline and months 1, 3, 6, and 12. For each lesion, time evolution of the phase rim, lesion volume, and T1 hypointensity were assessed. In autopsies of 3 progressive MS cases, the histopathology of the phase rim was determined.

RESULTS. In centripetal lesions, a phase rim colocalized with initial contrast enhancement. In 12 of 22 , this phase rim persisted after enhancement resolved. Compared with centripetal lesions with transient rim, those with persistent rim had less volume shrinkage and became more T1 hypointense between months 3 and 12 . No centrifugal lesions developed phase rims at any time point. Pathologically, persistent rims corresponded to an iron-laden inflammatory myeloid cell population at the edge of chronic demyelinated lesions.

CONCLUSION. In early lesion evolution, a persistent phase rim in lesions that shrink least and become more T1 hypointense over time suggests that the rim might mark failure of early lesion repair and/or irreversible tissue damage. In later stages of MS, phase rim lesions continue to smolder, exerting detrimental effects on affected brain tissue.

TRIAL REGISTRATION. NCT00001248.

FUNDING. The Intramural Research Program of NINDS supported this study.

\section{Introduction}

Myelin and oligodendrocytes are the major targets of a variety of immunological processes in multiple sclerosis (MS) (1). After myelin injury, oligodendrocyte precursor cells are recruited to the lesion site, where they mature, replace damaged oligodendrocytes, and remyelinate naked axons, thus limiting axonal degeneration and restoring saltatory conduction (2). Despite this potential, remyelination fails partially or completely in many MS lesions. Reasons for remyelination failure may include impaired oligodendrocyte precursor differentiation and maturation (3), axo-glial interaction (4), energy homeostasis, and clearance of cytotoxic inflammation (5-8). Among chronically demyelinated lesions, the pathologically described chronic active and slowly expanding (or "smoldering") lesions (9) might be considered the worst-case scenario of remyelination failure (10), with persistent inflammatory infiltration (macrophages/microglia) and evi-

Conflict of interest: The authors have declared that no conflict of interest exists. Submitted: December 23, 2015; Accepted: April 12, 2016.

Reference information: / Clin Invest. 2016;126(7):2597-2609. doi:10.1172/JCI86198. dence of ongoing demyelination at the lesion edge (11). However, in vivo, differentiation of such lesions from their "chronic inactive" counterparts remains elusive, in part because, unlike active lesions, chronic lesions do not display frank blood-brain-barrier abnormalities detectable on MRI by leakage of gadoliniumbased contrast agents.

A novel radiological finding in a subset of chronic lesions, the peripheral paramagnetic rim (12-17) that can be seen on magnetic resonance susceptibility imaging ( $\mathrm{T} 2^{*}$-weighted magnitude and susceptibility-weighted phase images), has been suggested to be a potential marker of chronic active lesions (12). However, against this hypothesis, a few short-term longitudinal MRI studies ( 1-2 years) failed to demonstrate that chronic rim lesions expand over time into the surrounding normal tissue $(16,18)$, although MRI studies with longer follow-up are still needed. Furthermore, existing radiological-pathological correlation studies have not resulted in a clear consensus on the extent to which the magnetic susceptibility-related MRI signal change in the lesion rim is caused only by iron deposition (iron-laden macrophages, ferritin, and/ or hemosiderin deposits) $(13,14,19,20)$ or by other MS-relevant 


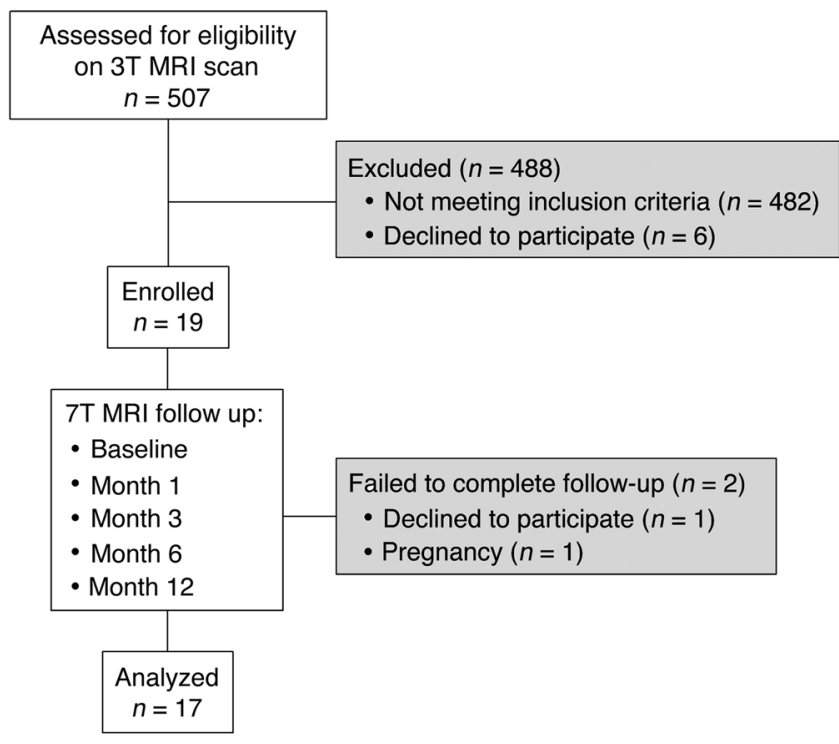

Figure 1. Flowchart summarizing MS patients' progress through the study.

pathological features, such as oxidative stress and disruption of tissue microarchitecture, including demyelination.

Establishing the onset of the phase rim and the fate of lesions with and without that feature, and interpreting such data within a comprehensive biological model of lesion development, are necessary to elucidate the rim's pathophysiological significance and potential relationship with mechanisms of lesion repair. In a crosssectional MRI study (16), coupling susceptibility-based imaging and dynamic contrast-enhanced (DCE) imaging at 7 tesla (7T), we showed that the rim is best appreciated on phase (rather than $\mathrm{T} 2^{*}$ ) images, that it may reflect the inflammatory edge (macrophages/microglia, their byproducts, and oxidative stress) of the newly forming lesion, and that it colocalizes with blood-brain-barrier opening in centripetally enhancing lesions $(16,21,22)$. As the phase rim is thus a potential endogenous biomarker for inflammation, we further hypothesized that (a) the centripetal contrast enhancement pattern and the related phase rim in active lesions are necessary for subsequent development of a rim in chronic lesions; and (b) lesions with a phase rim that persists after the resolution of contrast enhancement are those with unsatisfactory or delayed repair or perhaps ongoing tissue degeneration. To test this hypothesis, in 19 MS patients, we prospectively studied centrifugally (inside-out dynamic contrast leakage) and centripetally (outside-in dynamic contrast leakage) enhancing lesions at $7 \mathrm{~T}$ MRI at baseline and months (M) 1, 3, 6, and 12, focusing on the phase rim's presence and evolution as well as on lesion volume and T1-hypointensity changes over time. We performed exploratory peripheral cytokine/chemokine profiling at baseline and M12 and compared the results with patterns of lesion evolution (Supplemental Appendix; supplemental material available online

\section{Table 1. Main clinical, demographic, and MRI characteristics of the MS patients}

\begin{tabular}{|c|c|c|c|c|c|c|c|c|c|}
\hline Patient & Sex & Age (yr) & $\begin{array}{c}\text { Disease } \\
\text { duration (yr) }\end{array}$ & $\begin{array}{c}\text { Clinical } \\
\text { phenotype }\end{array}$ & $\begin{array}{l}\text { EDSS } \\
\text { score }\end{array}$ & $\begin{array}{l}\text { Disease-modifying } \\
\text { therapy (baseline/Y1) }\end{array}$ & $\begin{array}{l}\text { No. of contrast-enhancing } \\
\text { lesions (centrifugal/ } \\
\text { centripetal) }\end{array}$ & $\begin{array}{l}\text { No. of chronic } \\
\text { supratentorial lesions } \\
\text { at baseline }\end{array}$ & $\begin{array}{l}\text { No. of chronic lesions } \\
\text { with } T 2^{*} / \text { phase rim }{ }^{A}\end{array}$ \\
\hline 1 & $\mathrm{~F}$ & 39 & 8 & RR & 1 & None & $2(0 / 2)$ & 26 & $3 / 4(12 \% / 15 \%)$ \\
\hline 3 & $\mathrm{~F}$ & 38 & 8 & $\mathrm{RR}$ & 1.5 & Glatiramer acetate/None & $2(0 / 2)$ & 35 & $2 / 3(5 \% / 9 \%)$ \\
\hline 4 & $\mathrm{~F}$ & 60 & 0.2 & RR & 2 & None & $1(0 / 1)$ & 0 & $0(0 \%)$ \\
\hline 5 & $\mathrm{~F}$ & 37 & 9 & RR & 2.5 & IFN- $\beta$-1a/Natalizumab & $4(0 / 4)$ & 40 & $4 / 6(10 \% / 15 \%)$ \\
\hline 7 & $\mathrm{~F}$ & 33 & 0.2 & RR & 2 & None/IFN- $\beta-1 \mathrm{a}$ & $4(2 / 2)$ & 27 & $0 / 1(0 \% / 4 \%)$ \\
\hline 8 & $\mathrm{~F}$ & 38 & 6 & RR & 1.5 & None & $4(2 / 2)$ & 13 & $2 / 2(15 \% / 15 \%)$ \\
\hline 9 & M & 33 & 4 & RR & 1 & Clatiramer acetate & $1(0 / 1)$ & 34 & $8 / 9(24 \% / 27 \%)$ \\
\hline 10 & M & 37 & 1 & RR & 0 & None/Clatiramer acetate & $3(3 / 0)$ & 11 & $0 / 1(0 \% / 9 \%)$ \\
\hline 11 & $\mathrm{~F}$ & 34 & 1 & RR & 5 & None & $1(1 / 0)$ & 12 & $3 / 5(25 \% / 42 \%)$ \\
\hline 12 & $\mathrm{~F}$ & 37 & 19 & RR & 2 & None & $2(2 / 0)$ & 11 & 1/1 (9\%/9\%) \\
\hline 16 & $\mathrm{~F}$ & 42 & 2 & RR & 1.5 & None & $1(1 / 0)$ & 23 & $4 / 5(13 \% / 21 \%)$ \\
\hline 17 & $\mathrm{~F}$ & 37 & 1 & RR & 1.0 & Glatiramer acetate & $4(2 / 2)$ & 59 & $3 / 4(5 \% / 7 \%)$ \\
\hline
\end{tabular}

\begin{tabular}{|c|c|c|c|c|c|c|c|c|c|}
\hline \multicolumn{10}{|c|}{ Summary } \\
\hline $\begin{array}{l}17 \text { MS } \\
\text { patients }\end{array}$ & F/M & $\begin{array}{l}\text { Mean } \\
\text { age } \\
\text { (SD) }\end{array}$ & $\begin{array}{l}\text { Mean disease } \\
\text { duration (SD) }\end{array}$ & $\mathrm{RR} / \mathrm{SP}$ & $\begin{array}{l}\text { Median } \\
\text { EDSS } \\
\text { (range) }\end{array}$ & $\begin{array}{l}\text { No. of untreated } \\
\text { patients }\end{array}$ & $\begin{array}{l}\text { Median no.(range) of } \\
\text { contrast-enhancing lesions }\end{array}$ & $\begin{array}{l}\text { Median no. (range) } \\
\text { of supratentorial } \\
\text { chronic lesions }\end{array}$ & $\begin{array}{c}\text { Median no. (range) } \\
\text { of chronic lesions } \\
\text { with phase rim }\end{array}$ \\
\hline & $15 / 2$ & 40.9 & $6.5(6.4)$ & $16 / 1$ & $1.5(1-5.5)$ & 8 of 17 & $2(1-5)$ & $21(0-59)$ & $2(1-9)$ \\
\hline
\end{tabular}

RR, relapsing remitting; SP, secondary progressive; EDSS, Expanded Disability Status Scale; Y1 = year $1 .{ }^{A}$ Discrete supratentorial chronic lesions as visible on 50 axial 1-mm slices. 
A

Persistent phase rim after enhancement resolution

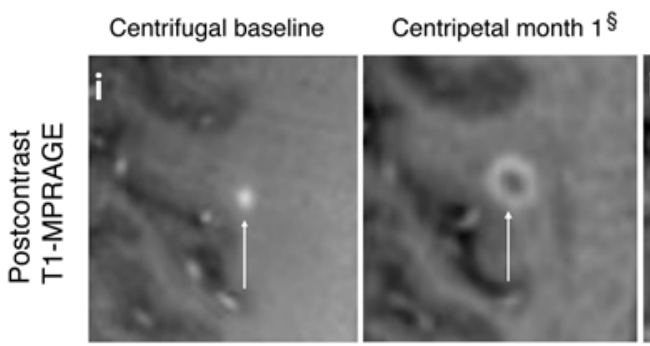

Month 3

Month 6

Month 12

Month 18
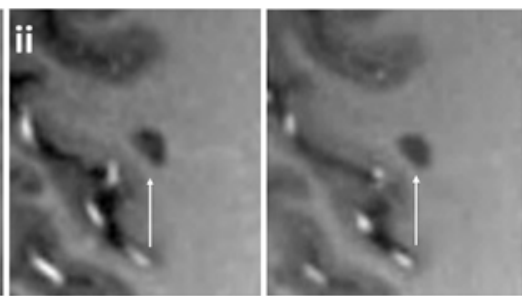

$+4$
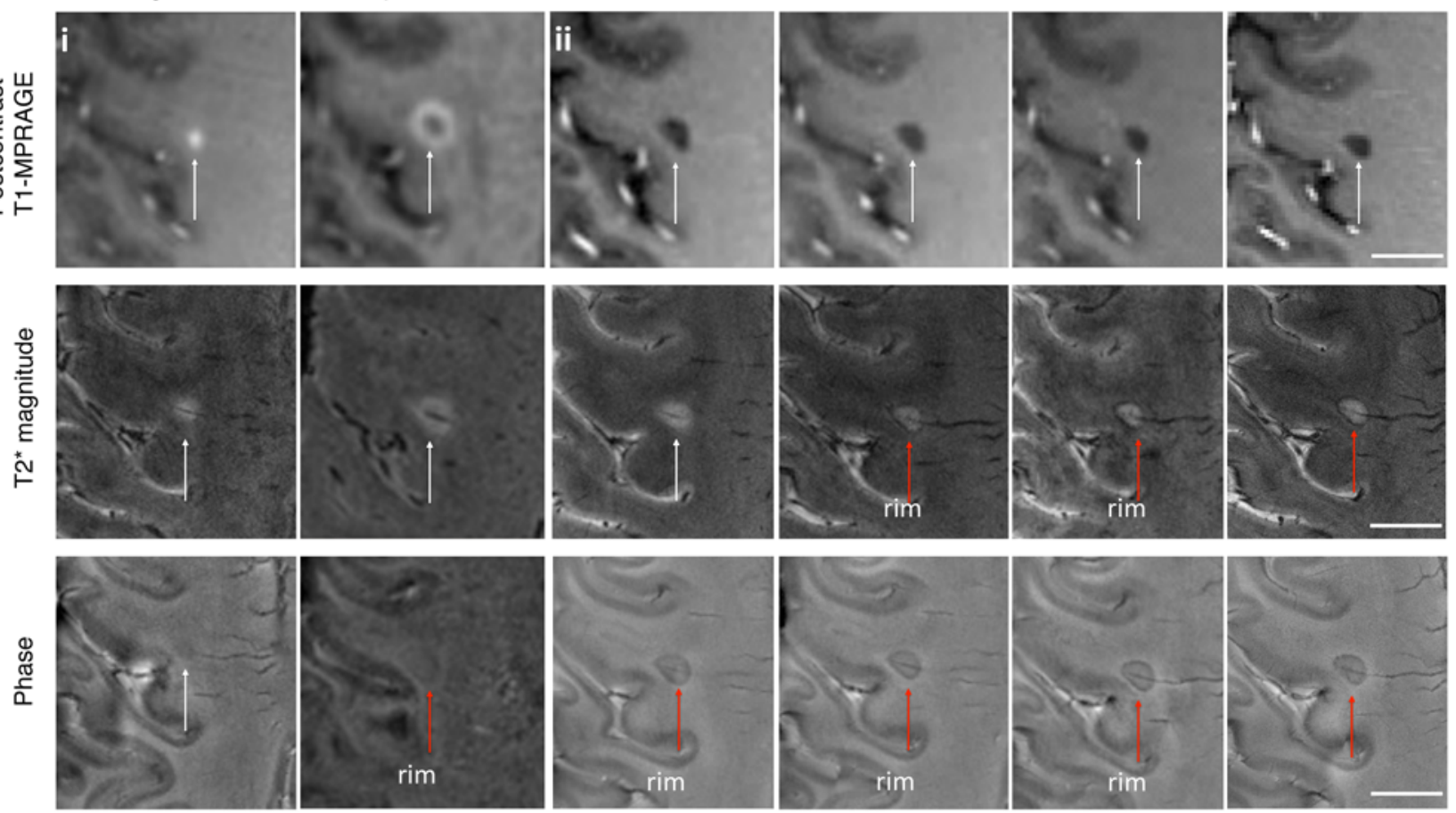

B

Transient phase rim after enhancement
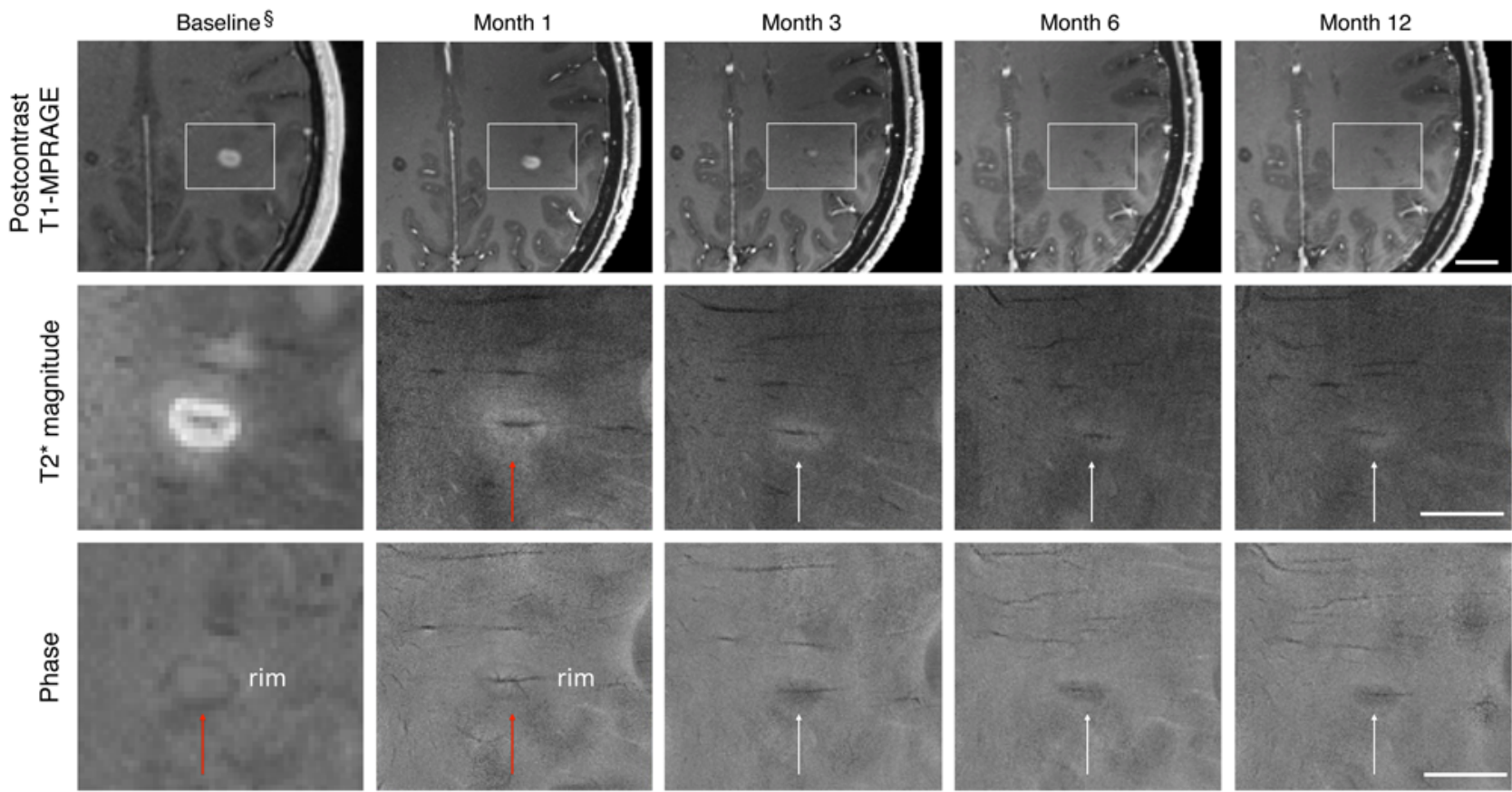

Figure 2. Centripetal lesions: persistent versus transient phase rim during follow-up. (A) Persistent phase rim after enhancement resolution: Eighteenmonth longitudinal evolution of a centripetally enhancing MS lesion with phase rim at 7T MRI in a 49-year-old woman with secondary progressive MS (Expanded Disability Status Scale 5.5, disease duration 19 years). (i) Postcontrast T1-weighted images capture the shift from centrifugal enhancement at baseline to centripetal enhancement at M1. When the lesion enhances centripetally, a hypointense rim on noncontrast phase images colocalizes with initial opening of the blood-brain barrier in peripheral vessels. (ii) After resolution of enhancement (M3, M6, M12, and M18), the rim persists on phase images (red arrows) and appears also on T2*-weighted magnitude images at M6, M12, and M18. (B) Transient phase rim after enhancement resolution: Twelvemonth longitudinal evolution of a centripetally enhancing MS lesion with phase rim at 3T and 7T MRI in a 38-year-old woman with relapsing-remitting MS (Expanded Disability Status Scale 1.5, disease duration 6 years). In this case, as well, a hypointense rim can be discerned at baseline on the noncontrast phase images (red arrows); however, the rim disappears in the months following enhancement resolution. ${ }^{\S}$ Scans acquired at 3 T MRI. Scale bar: 10 mm. 
Table 2. One-year longitudinal evolution of the phase rim, lesion volume, and T1 hypointensity in centrifugally and centripetally enhancing MS lesions

\begin{tabular}{|c|c|c|c|}
\hline & \multirow{2}{*}{$\begin{array}{l}\text { Exclusively centrifugal } \\
\text { DCE pattern }\end{array}$} & \multicolumn{2}{|c|}{ Centripetal DCE pattern } \\
\hline & & Transient phase rim & Persistent phase rim \\
\hline No. of lesions & 20 & 10 & 12 \\
\hline \multicolumn{4}{|c|}{ Paramagnetic rim on $\mathrm{T}^{*}$ (number of lesions) } \\
\hline Baseline & 0 & 4 & 9 \\
\hline M12 & 0 & 0 & 12 \\
\hline \multicolumn{4}{|c|}{ Mean lesion volume (SD) (mm³) } \\
\hline Baseline $^{A}$ & $27(17)$ & 168 (151) & $664(823)$ \\
\hline M3 & $18(12)$ & $138(136)$ & $313(304)$ \\
\hline M6 & $13(11)$ & $86(69)$ & $243(208)$ \\
\hline M12 & 11 (11) NA in 3 lesions & 72 (72) NA in 1 lesion & 206 (204) NA in 1 lesion \\
\hline \multicolumn{4}{|c|}{ Percentage of lesion volume shrinkage (SD) } \\
\hline (Baseline - M12)/baseline & $64(16)$ & $56(13)$ & $54(15)$ \\
\hline (M3 - M12)/M3 & $39(20)$ & $41(11)$ & $23(15)$ \\
\hline \multicolumn{4}{|c|}{ Mean T1-hypointensity values (SD) } \\
\hline Baseline $^{A}$ & $-17(7)$ & $-26(10)$ & $-36(13)$ \\
\hline M3 & $-17(6)$ & $-17(4)$ & $-26(7)$ \\
\hline M6 & $-17(7)$ & $-16(6)$ & $-28(7)$ \\
\hline M12 & $-15(8)$ & $-17(5)$ & $-28(9)$ \\
\hline \multicolumn{4}{|c|}{ Percentage of intralesional T1 intensity change } \\
\hline (Baseline - M12)/baseline & $10(58)$ & $33(19)$ & $8(29)$ \\
\hline$(\mathrm{M3}-\mathrm{M12}) / \mathrm{M3}$ & $8(32)$ & $1(13)$ & $-17(21)$ \\
\hline \multicolumn{4}{|c|}{ Percentage of lesions with reduced T1 intensity over time } \\
\hline M3 to M12 & 35 & 11 & 70 \\
\hline \multicolumn{4}{|l|}{ Lesion brain location } \\
\hline No. of lesions, JC/PV/DWM & $7 / 3 / 10$ & $1 / 4 / 5$ & $8 / 1 / 3$ \\
\hline
\end{tabular}

with this article; doi:10.1172/JCI86198DS1). Finally, we assessed the histopathological correlates of the phase rim in the formalinfixed brains of 3 individuals with progressive MS to support part of the model of lesion development and rim evolution that we propose based on in vivo data.

\section{Results}

From April 2012 to April 2015, we recruited 19 people with MS (18 relapsing-remitting and 1 secondary-progressive with relapses) under an IRB-approved natural history protocol (Figure 1). Only patients untreated or treated with a first-line therapy (glatiramer acetate, IFN- $\beta$, dimethyl fumarate) at baseline were included (Table 1). Based on the identification of new enhancing supratentorial lesions in scheduled examinations at 3T (Supplemental Table 1), participants were invited to undergo a prospective longitudinal 7T MRI study at baseline and M1, M3, M6, and M12. During the study period, 125 scans at 7T MRI were performed. Two people with a relapsing-remitting disease course dropped out and were not included in the final analysis (Figure 1). Experienced MS clinicians determined disability according to the Expanded Disability Status Scale (23) and obtained clinical data at baseline and M12. Demographic and clinical information is provided in Table 1.
In vivo radiological results

All participants but 2 had at least 1 chronic lesion with phase rim at baseline (median: 2 lesions, range: 1-9, Table 1), representing 14\% (median: $9 \%$, range: $4 \%-42 \%$ ) of supratentorial discrete chronic lesions. On postcontrast T1-weighted magnetization-prepared rapid gradient echo (T1-MPRAGE), we detected and followed 42 discrete supratentorial enhancing lesions (longest lesion axis $\geq 1.5 \mathrm{~mm}$ ). Twenty lesions showed exclusively centrifugal enhancement (hereafter termed "centrifugal lesions"), and 22 showed centripetal after initial centrifugal enhancement ("centripetal lesions"). The median number of enhancing lesions per participant was 2 (range: $1-5$ ).

Longitudinal peripheral phase rim assessment. At baseline, in centripetal lesions, the phase rim colocalized with the initial site of contrast enhancement (rim thickness mean \pm SD: $465 \pm 133 \mu \mathrm{m})$. During the follow-up, in 10 of 22 centripetal lesions, the rim disappeared within 3 months after enhancement resolved; in the remaining 12 centripetal lesions, the phase rim persisted (Figure 2).

In centripetal lesions with persistent rim, the mean phase rim thickness was stable over time (baseline: $498 \pm 160$, M3: $627 \pm$ 119, M6: $646 \pm 189$, M12: $536 \pm 86 \mu \mathrm{m}$ ), but in 3 lesions the rim became increasingly evident on $\mathrm{T} 2{ }^{*}$-weighted magnitude images (9 of 12 lesions had a T2* rim at baseline; Table 2 ). As shown in Table 2, centripetal lesions with persistent phase rim were more frequently found in the cortical/juxtacortical region than in the periventricular and deep white matter regions. During the follow-up, in some cortical/juxtacortical lesions, the phase rim was qualitatively more prominent at the white/gray matter junction than in the deep white matter (Supplemental Figure 1). By comparison, none of the 20 centrifugal lesions developed a phase rim at any time point (Figure 3).

Longitudinal lesion volume assessment. The mean lesion volume was higher at all time points in centripetal lesions with persistent rim compared with the other 2 groups (Table 2). The trajectory of volume over time for each lesion is shown in Figure $4 \mathrm{~A}$. The overall percentage of lesion volume shrinkage from baseline to M12 was similar across the 3 lesion groups, but comparing M3 (after resolution of initial lesion edema) and M12, the relative volume shrinkage of centripetal lesions with persistent phase rim was least $(P=0.028$, Table 2 and Figure $4 \mathrm{~B})$. In Figure 4A, it is also evident that participants who developed persistent-rim lesions were older than those who developed 

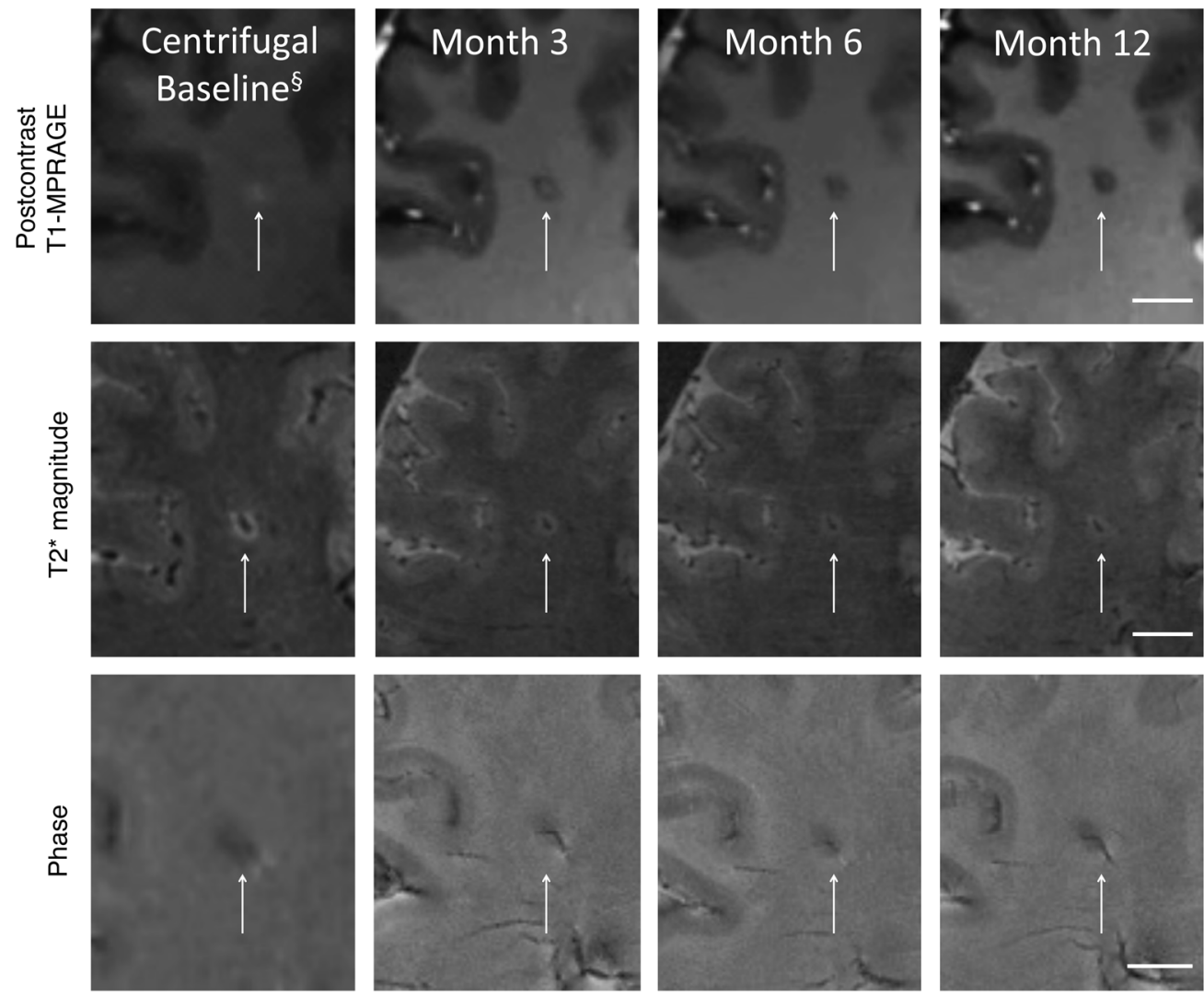

Figure 3. Fate of centrifugal lesions. Twelve-month longitudinal evolution of a centrifugally enhancing MS lesion at 3T and 7T MRI in a 58-year-old woman with relapsing-remitting MS (Expanded Disability Status Scale 1.5, disease duration 11 years) that arises from a clearly visible central vein. A phase rim is not discerned at any time point. After closure of the blood-brain barrier, the lesion is visible as hypointense on phase images and hyperintense on T2* ${ }^{*}$ weighted magnitude images. ${ }^{\S}$ Scan acquired at 3T MRI. Scale bar: $10 \mathrm{~mm}$.

transient-rim lesions (mean ages 44.3 and 34.7 years, respectively, $P=0.04$, ANOVA).

Longitudinal lesion $T 1$ intensity assessment. The mean normalized T1-weighted signal intensity was lower at all time points in persistent-rim lesions compared with the other 2 lesion groups (Table 2$)$. Overall, partial recovery of $\mathrm{T} 1$ intensity $(-25 \%)$ was seen from baseline to M12 across the 3 groups. However, rim persistence was associated with progressively lower $\mathrm{T} 1$ intensity between $\mathrm{M} 3$ and M12, which was seen in 7 of 10 lesions with persistent rim (70\%) compared with 7 of 26 lesions without rim (27\%) $(P=0.03$, Fisher's exact test) (Figure 4C). A schematic representation of the 3 scenarios of lesion development and evolution according to status of the blood-brain barrier, phase rim, lesion volume, and lesion T1 hypointensity is shown in Figure 5.

Prediction of phase rim persistence after 1 year. Among clinical and radiological variables (participant age, brain location, and baseline presence of a $\mathrm{T} 2^{*}$ rim, lesion volume, and $\mathrm{T} 1$ intensity), a $\mathrm{T}^{*}$ rim and lesion volume at baseline strongly predicted the persistence of a phase rim at 12 months. According to these 2 variables, and irrespective of the initial contrast enhancement pattern, a persistent phase rim is expected when (a) the baseline lesion volume is $\geq 224 \mathrm{~mm}^{3}$ in the absence of a $\mathrm{T}^{*}$ rim at baseline (sensitivity $100 \%$, specificity $92 \%$ ); or (b) the baseline lesion volume is $\geq 162 \mathrm{~mm}^{3}$ in the presence of a T2* rim at baseline (sensitivity $86 \%$, specificity $100 \%)$.

\section{Clinical correlations}

Three participants had centripetal lesions with both transient and persistent phase rims. The only participant with secondary progressive MS who was enrolled in this cohort had 2 centripetally enhancing lesions, both of which had a persistent phase rim. Five of 17 participants had neurological symptoms at the time of enrollment and were treated with i.v. steroids after the baseline MRI scan. Interestingly, steroid administration was associated with lower chance of a phase rim at 12 months (1 of 14 treated lesions [7\%] compared with 11 of 28 untreated lesions [39\%], $P=0.036$ ).

\section{Neuropathological correlation}

In an autopsy case, 5 MS lesions ( 3 periventricular and 2 leukocortical) with persistent phase rim, seen on both in vivo and postmortem 7T MRI, were completely demyelinated, without evidence of complete or partially remyelinated white matter areas (Figure 6 and Supplemental Figure 3). The lesion edges, where the phase rims were detected on MRI, were characterized by the extensive presence of a CD68-positive demyelinating inflammatory infiltrate (comprising macrophages and/or activated microglia, Figures 6, 7, 8 and Supplemental Figure 3) that tended to distribute in the periplaque along some of the veins crossing the lesion edge. These lesions were found to expand on in vivo 7-year longitudinal MRI data. The majority of CD68-positive cells closer to the lesion edge showed intracellular iron accumulation (double staining with anti-CD68 and DAB-Turn- 

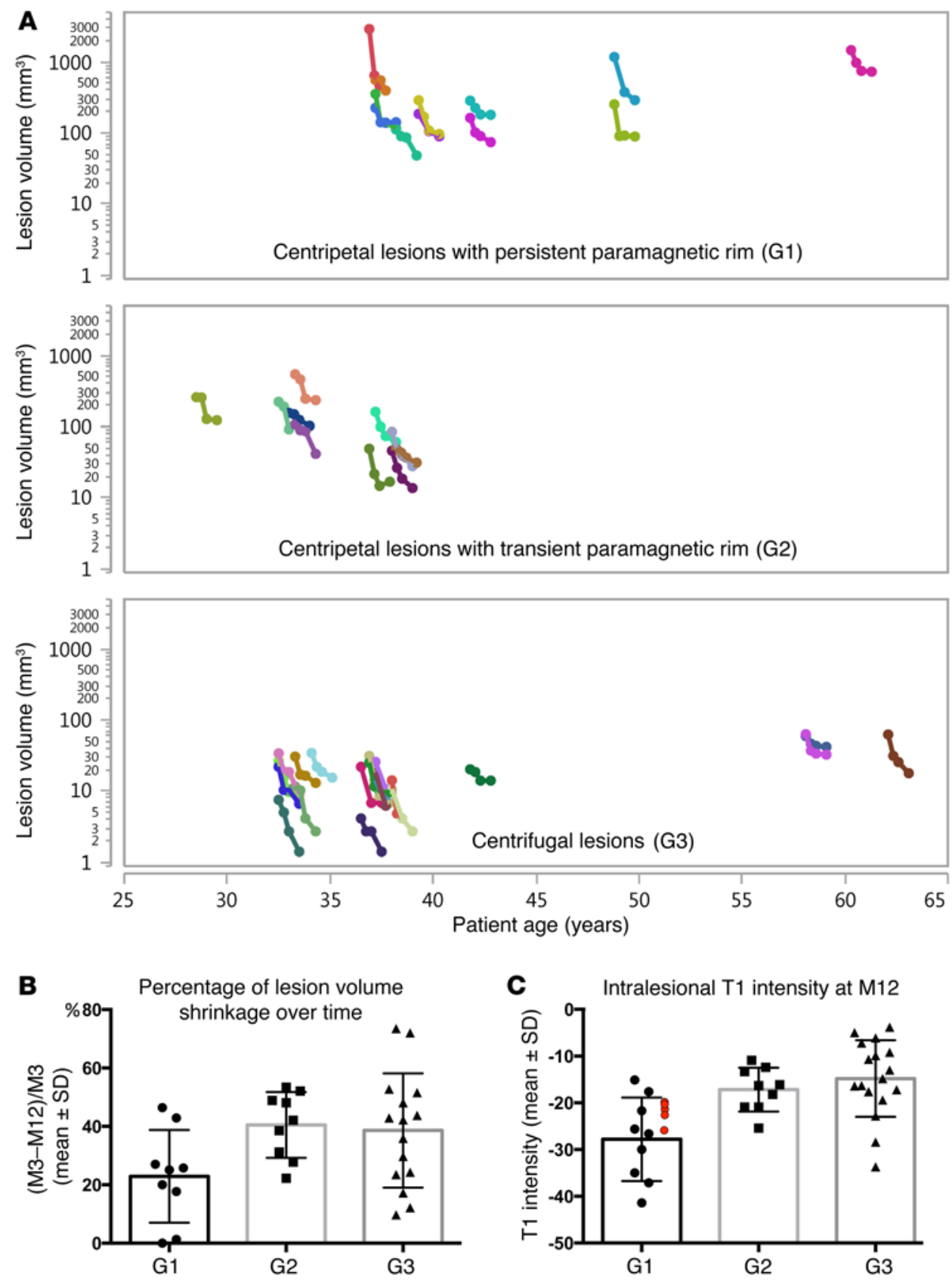

Figure 4. Longitudinal lesion volume and T1-hypointensity assessment. (A) Semilogarithmic plot of the longitudinal volume for each lesion according to participant age and lesion group (G1, C2, G3). Three main observations about centripetal lesions with persistent phase rim derive from this graph: (a) their lesion volume shrinkage over time tends to plateau after the first 3 months; (b) their mean lesion volume is higher at all time points; and (c) compared with centripetal lesions with transient phase rims, they tend to occur in older individuals. (B) Percentage of lesion volume shrinkage (mean \pm SD) between M3 and M12 for each lesion group. (C) Lesional T1 hypointensity at M12 for each lesion group (mean \pm SD; $\mathrm{T} 1$ signal intensity is expressed in units of SD of normal-appearing white matter signal). In G1, red dots show the in vivo mean T1 hypointensity of the 5 pathologically assessed demyelinated lesions with phase rim. G1: centripetal lesions with persistent phase rim; C2: centripetal lesions with transient phase rim; C3: centrifugal lesions. bull, Figures 7 and 8). CD8-positive T lymphocytes were mostly detected in perivascular spaces of small veins and capillaries at the lesion edge (CD8/CD68 ratio ranging from 0.03 to 0.23 at the lesion edge), while CD4- and CD20-positive perivascular lymphocytes were extremely rare. Non-iron-laden reactive astrocytes were seen at both lesion edge and lesion center (double staining with anti-GFAP and DAB-Turnbull, Figure 7). Small foci of perivenular demyelination and myelin rarefaction were visible in the periplaque zone. Myelin PLP-positive oligodendrocytes and oligodendrocyte precursor cells $\left(\mathrm{OLIG}^{+} / \mathrm{ASPA}^{-}\right)$were prevalent in the cortical portions of leukocortical lesions but rarely at the white matter lesion edge (Figure 7).

Extensive axonal loss and transection, as well as impairment of axonal transport (SMI32 nonphosphorylated neurofilament staining) in remaining axons, were seen throughout the center of all lesions, corresponding to in vivo mean intralesional T1 inten- sity values ranging from -25.1 to -18.7 (fully consistent with longitudinal in vivo data on centripetal lesions with persistent rim; see Table 2 and red dots in Figure 4C). At the lesion edge, axons were progressively better preserved and fiber bundles more clearly discerned (Figure 6), even if sparse end terminals of degenerating axons (24) were seen on SMI32 (Figure 7).

In 2 other autopsy MS cases, 6 non-phase rim lesions (4 periventricular, 1 in the deep white matter, and 1 juxtacortical) did not show a CD68-positive inflammatory infiltrate at the lesion edge, but there were varying degrees of intralesional non-iron-laden CD68-positive infiltrates (Figure 8 and Supplemental Figure 4).

\section{Discussion}

In this study, newly forming supratentorial MS lesions were classified according to their pattern of dynamic contrast enhance- 


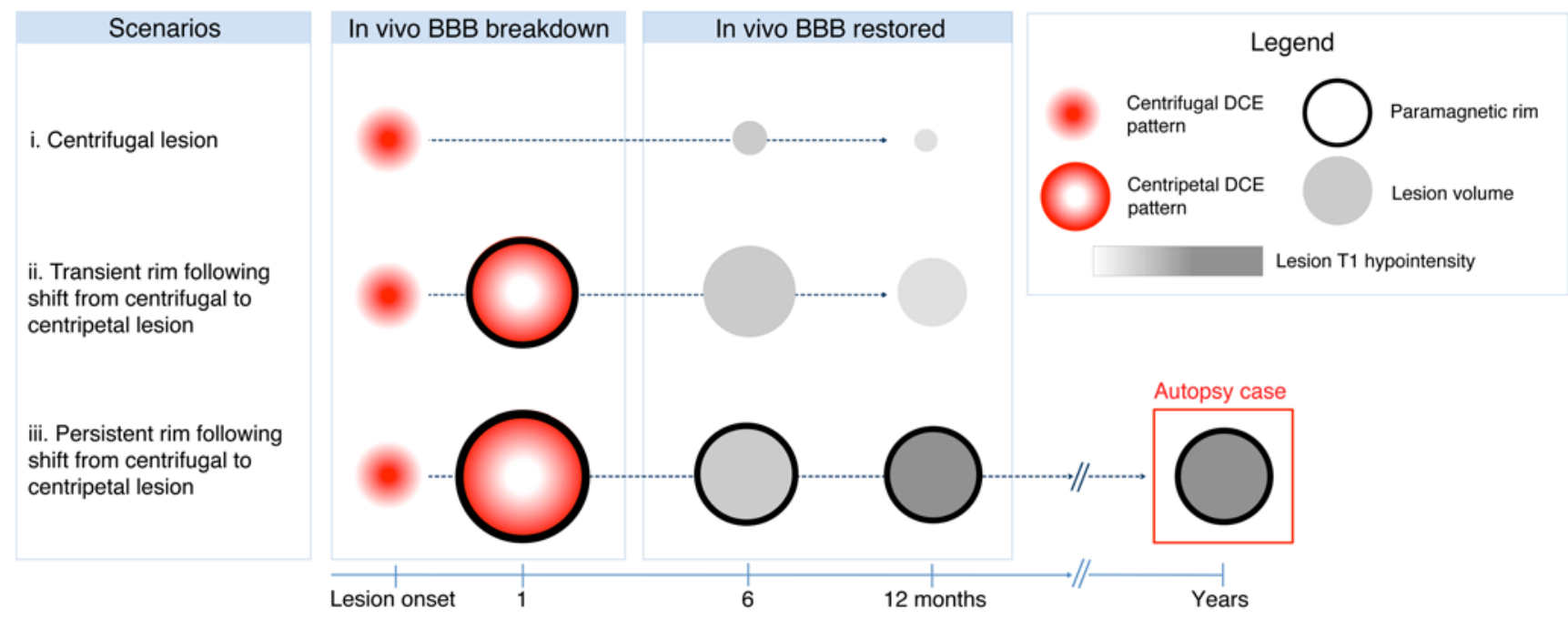

Figure 5. Graphical representation of the 3 scenarios of lesion development and evolution according to status of the blood-brain barrier, phase rim, lesion volume, and lesion T1 hypointensity. (i) Evolution of a fully centrifugal lesion: no shift to centripetal enhancement pattern; no phase rim at any time point. (ii) Evolution of a centrifugal lesion to a centripetal lesion with transient phase rim: disappearance of the phase rim during the follow-up. (iii) Evolution of a centrifugal lesion to a centripetal lesion with persistent phase rim: persistence of the phase rim after enhancement resolution and reduced lesion volume shrinkage and reduced $\mathrm{T} 1$ intensity over time in comparison with the other lesion types. Years from lesion onset, the pathological correlate of the persistent phase rim can be assessed at autopsy. BBB, blood-brain barrier; DCE, dynamic contrast enhancement imaging.

ment $(16,21,22)$ (centrifugal or centripetal) and followed over at least 1 year to assess, in vivo, the complex interplay among bloodbrain-barrier status, inflammation, and lesion evolution. The histopathological correlate of the phase rim was also described (Figures 6-8) in order to support a proposed phenomenological model of lesion development and rim evolution that derives from the in vivo data (Figure 9).

According to a commonly accepted model of MS lesion development, after a myelin antigen is encountered, demyelination within a newly forming lesion proceeds outward from a small central vein. This process, which involves disruption of the bloodbrain barrier, is mirrored in the centrifugal pattern of contrast enhancement by DCE MRI $(21,22)$. The brain's natural response is to contain this destructive inflammatory process through a combination of immune-mediated mechanisms and astroglial reaction. In some active lesions, for unknown reasons, failure of early tissue-protective mechanisms can lead to more exuberant lesion growth, which triggers a stronger immune reaction at the lesion edge that may isolate the lesion from surrounding unaffected parenchyma. Our previous studies $(16,21,22)$, interpreted in the context of prior work on the CNS injury response (7), suggest that this process involves opening of the blood-brain barrier in capillaries at the lesion edge. Such opening manifests as a centripetal pattern of contrast enhancement by DCE MRI. The attendant infiltration of blood-derived monocytes/macrophages, and/or activation of resident microglia, with their related by-products, is reflected in an early phase rim. The specific paramagnetic substances that underlie the early phase rim are not yet determined.

In centripetal lesions, our current imaging data show that 2 possible scenarios can play out (Figures 2 and 5). In approximately half of the lesions, the early phase rim persists, and we hypothesize that this represents ongoing inflammation despite closure of the blood-brain barrier as detected on gadolinium-enhanced MRI.
In this scenario, the persistent pathological process corresponding to the phase rim is likely to be related to the high ratio of inflammatory (iron-laden, ref. 25; and hence paramagnetic, Figure 8) to antiinflammatory/repair-promoting myeloid cells at the lesion edge, but also possibly to persistent oxidative stress. Notably, the identities and functional phenotypes of macrophages have been shown to be extremely dynamic and responsive to varying environmental signals, and an intermediate activation status is often detected (26). Thus, we have chosen to use the broader terms "inflammatory" and "repair-promoting" (antiinflammatory) in lieu of M1 and M2 polarization status (27). As in other inflammatory diseases $(7,28)$, the shift from the initially prevalent population of proinflammatory to antiinflammatory cells (29), and the subsequent prompt clearance of the proinflammatory population, may have a fundamental role in promoting tissue remodeling (resolution of the glial scar) and enhancing repair (remyelination, new synapse sprouting) (30-32) (Figure 9). We propose that this is the case in the second scenario, in which the phase rim disappears when enhancement resolves (here, we termed these lesions "centripetal lesions with transient rim").

The analysis of the trajectory of lesion volumes and T1 hypointensity over time suggests that the phase rim is not only a marker of persistent inflammation within the first year of lesion development but also a possible marker of impaired or delayed lesion repair and irreversible tissue degeneration. In the absence of reliable and direct imaging markers of repair/remyelination, tracking of the longitudinal lesion signal intensity (in terms of its return toward normal white matter signal) and volume is at present the established strategy to assess short-term lesion repair $(33,34)$. In this study, two major imaging findings characterize the evolution of persistent-rim lesions in comparison with the other two scenarios of lesion development: (a) lower rate of lesion volume reduction at each time point, and (b) progressive lowering of T1 signal inten- 
A

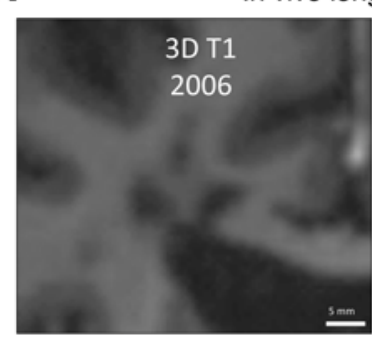

In vivo longitudinal MRI

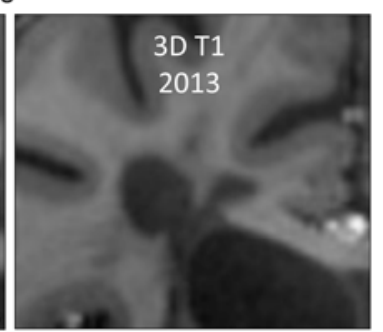

\section{Postmortem 7T MRI}

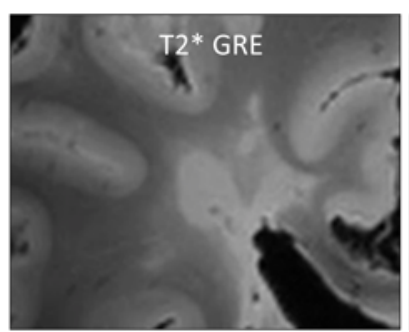

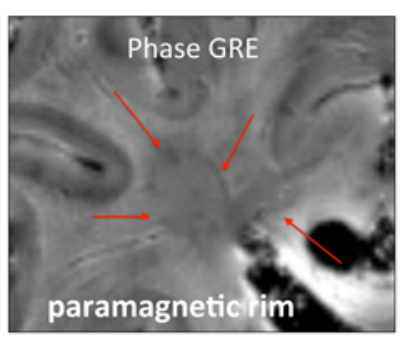
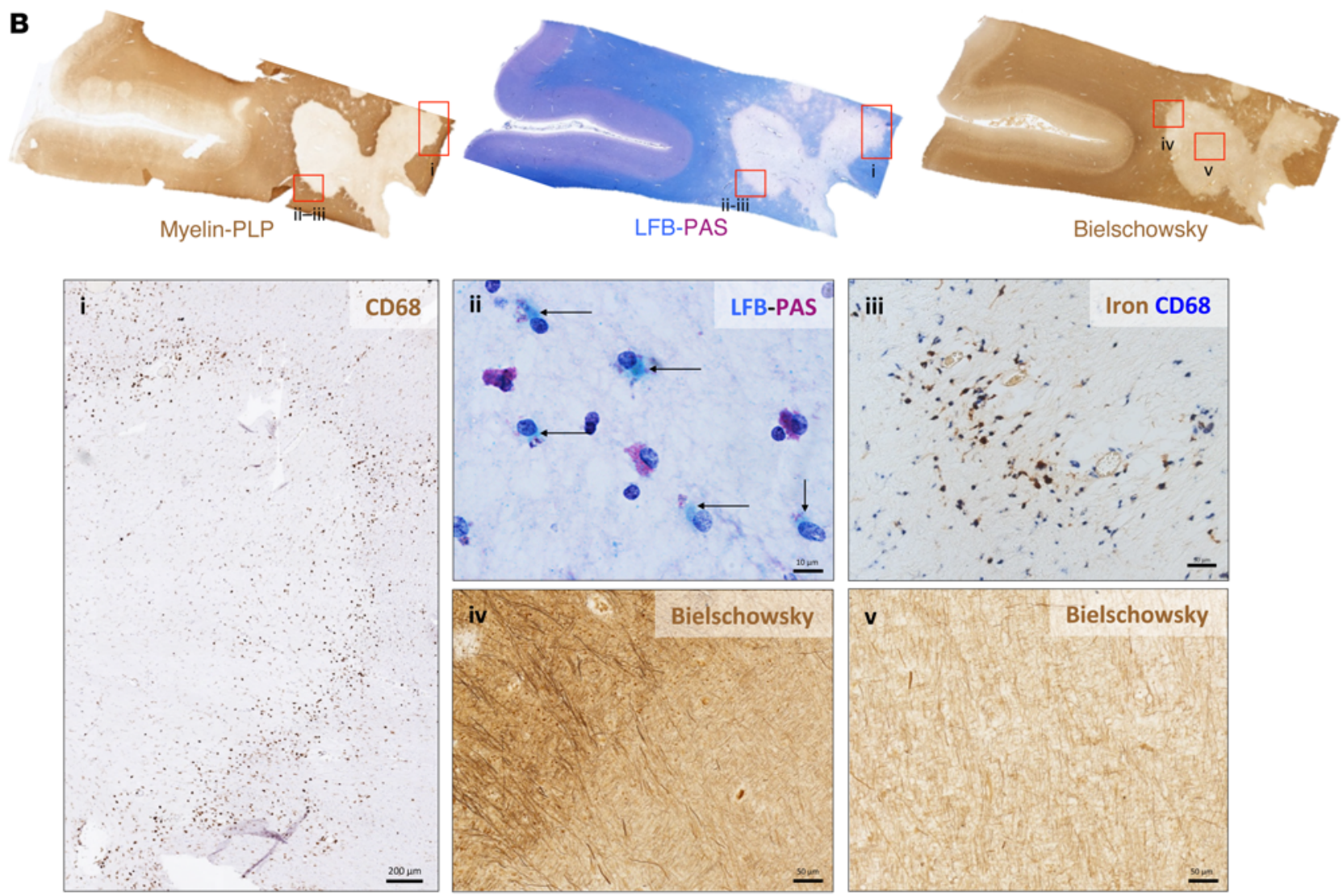

Figure 6. MRI/pathology of demyelinated lesions with persistent phase rim. Images shown are from lesions 2 and 3 in Figure 8 . (A) In vivo and postmortem 7T MRI shows 2 periventricular lesions with persistent phase rims that become partially confluent over time as the lesion expands (between 2006 and 2013). The rims were visible on an in vivo 7T MRI in 2013 (not shown), as well as postmortem (red arrows). On the in vivo 7T 3D T1-MPRAGE (2013), these lesions appear strongly hypointense (mean lesion T1 intensity values, respectively: -25.1 and -21.2 in units of SD of normal-appearing white matter signal; mean cerebrospinal fluid T1 intensity: -29.9). Scale bar: $5 \mathrm{~mm}$. (B) In vivo and postmortem MRI-guided histopathology allowed precise localization of the target area. MRI-matched thumbnails of representative serial sections (10- $\mu \mathrm{m}$-thick sections) show the Luxol fast blue/periodic acid-Schiff (LFB-PAS) stain for myelin, myelin proteolipid protein (PLP) immunohistochemistry, and Bielschowsky staining for axons. Insets i-v are indicated as red squares on the thumbnails to facilitate their localization and the interpretation of the pathological data. Both lesions were completely demyelinated. (i-iii) The lesion edge, where the phase rim was detected on MRI, is characterized by the presence of an extensive CD68-positive cellular infiltrate, corresponding to macrophages/activated microglia (inflammatory infiltrate thickness $~ 200-400 \mu \mathrm{m}$ ). Luxol fast blue-positive myelin debris (cyan, black arrows) and late myelin degradation products (lipofuscin, purple) can be seen within macrophages at the lesion edge, suggesting ongoing demyelination (ii). The majority of CD68-positive cells also stained positive by the DAB-Turnbull method, indicating the intracellular accumulation of iron (iii). (iv and v) Extensive axonal loss with transection and dystrophy of the remaining axons is seen throughout the lesion center (v). At the lesion edge, some axons were better preserved, and fiber bundles could be discerned (iv). Scale bars: $200 \mu \mathrm{m}$ (i); $10 \mu \mathrm{m}$ (ii); $50 \mu \mathrm{m}$ (iii-v).

sity between M3 and M12 (Figure 4 and Table 2). Relevantly and as confirmed at autopsy, lesional T1 hypointensity has been shown to associate with the degree of axonal loss in correlative radiologypathology studies (35-38), and resolving T1 hypointensity has been suggested as a potential marker of remyelination (38). Among the possible explanations for failure of repair and/or remyelination in persistent-rim lesions, in the context of a preponderance and persistence of myeloid cells (pathological data and proposed phenomenological model shown in Figures 6-9), are inhibition of oligodendrocyte precursor cell recruitment and maturation, impairment of normal axo-glial interactions, disturbance of mitochondrial function in both oligodendrocyte precursors and axons, and cytotoxic- 

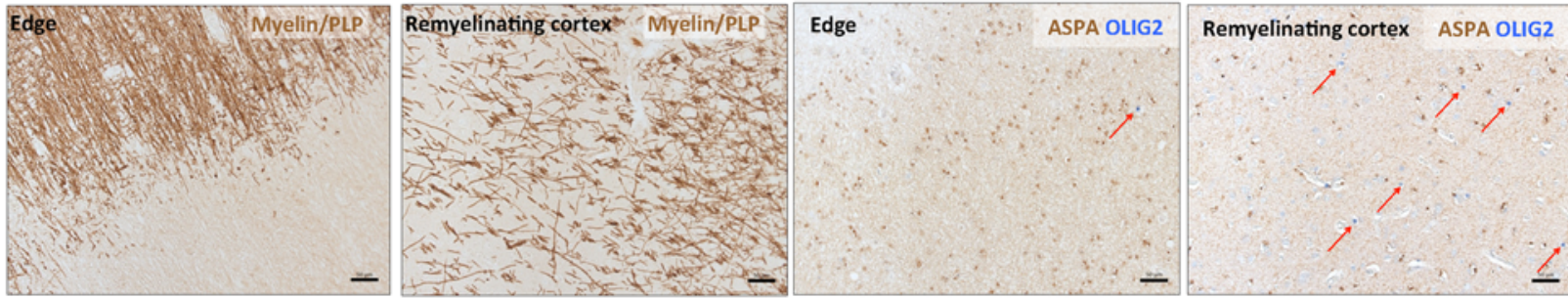

Macrophages/activated microglia colocalize with the phase rim at the lesion edge

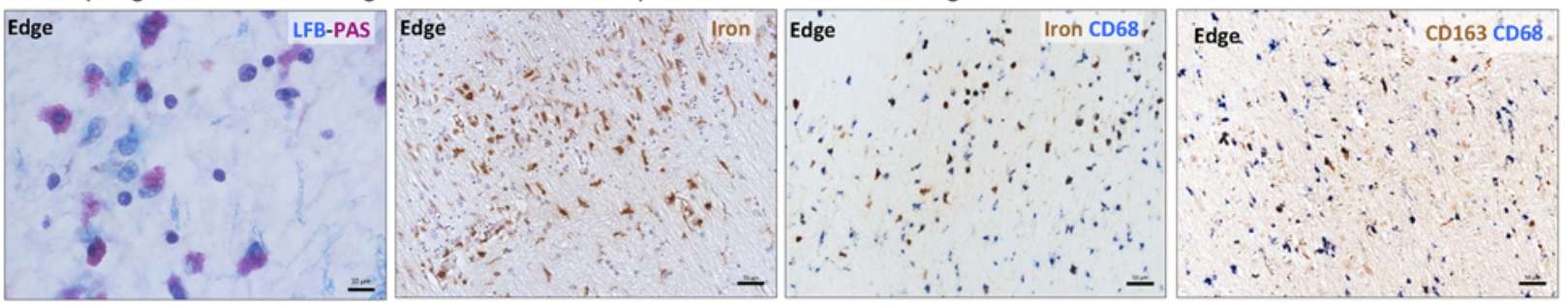

Reactive astrocytes and axonal damage
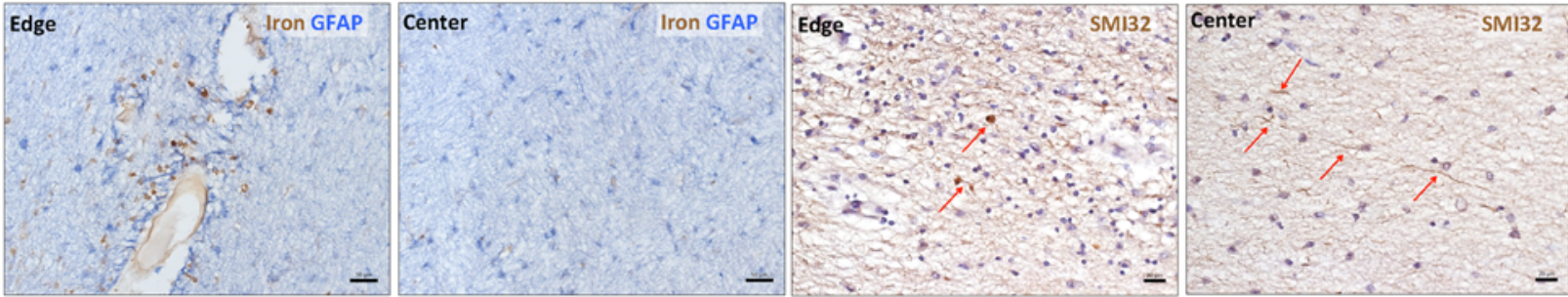

Figure 7. Histological features of MS lesions with phase rim. Chronic demyelination colocalizes with the phase rim at the lesion edge. There are no signs of ongoing remyelination and only rare oligodendrocyte precursor cells (OLIG2+/ASPA- cells, red arrows). In a cortical lesion, on the other hand, there is evidence of oligodendrocyte precursor cells as well as remyelination. Macrophages/activated microglia also colocalize with the phase rim at the lesion edge. Luxol fast blue (cyan) and lipofuscin (purple) inclusions within these cells suggest ongoing early and late myelin degradation process ( $\times 100$ magnification) A rim of iron-laden CD68-positive cells are clearly present at the lesion edge (DAB-Turnbull staining alone and double staining with anti-CD68/DAB-Turnbull). Mature tissue macrophages expressing the scavenger receptor CD163 were also represented in the CD68 population, suggesting that the population was not homogeneous (double staining with CD68/CD163). Reactive astrocytes and axonal damage: Non-iron-laden reactive astrocytes were seen in the demyelinated lesion center and demyelinating lesion edge (double staining with GFAP/DAB-Turnbull). Residual axons within the lesion center showed evidence of impaired axonal transport (positive staining for nonphosphorylated neurofilaments expressing SMI32). Red arrows indicate the presence, at the lesion edge, of sparse SMI32-positive ovoids suggesting ongoing axonal degeneration. Scale bars: $50 \mu \mathrm{m}$ (top row); $10 \mu \mathrm{m}$ (left panel, middle row); 50 $\mu \mathrm{m}$ (right 3 panels, middle row); $50 \mu \mathrm{m}$ (left 2 panels, bottom row); $20 \mu \mathrm{m}$ (right 2 panels, bottom row).

ity (3-8). Indeed, inflammatory mediators such as free radicals or nitric oxide, which are also commonly paramagnetic, can in principle persist at the lesion edge, stimulating a vicious circle of detrimental inflammation and worsening neurodegeneration. Such a cycle may be akin to the recently described "unrestrained macrophage activation" in nonhealing wounds, which bear important similarities to poor response to CNS injury (7).

In our pathological samples, the most striking histological feature of chronic phase rim lesions is the presence of CD68-associated smoldering demyelination at the lesion edge that allows their pathological classification as "chronic active"/"slowly expanding"/"smoldering" lesions. The interpretation of our in vivo MRI data and postmortem findings (Figure 7) is, therefore, not in conflict with the pathological finding of iron accumulation within a subset of macrophages/activated microglia at the edge of chronic phase rim lesions $(13,14,19,20,25,39)$, even if other relevant factors, such as oxidative stress and disruption of the microstructural organization of the tissue (39-42), may also affect the phase signal in active lesions. The role of iron in such lesions is complex, as iron can in principle both enhance neurodegeneration and stimulate remyelination $(43,44)$. Variable amounts of free iron are released during the process of demyelination. This iron may be rapidly taken up by myeloid cells and contribute to the maintenance of their polarization (25); it may then be released, bound to ferritin, for use in oligodendrocyte precursors (45-47). In cells of the oligodendroglial lineage, several enzymes, including those involved in mitochondrial function, differentiation, and myelin formation, are iron-dependent $(46,47)$.

Interestingly, in 3 of 12 centripetal lesions with persistent phase rim, we also detected the appearance of a rim on $\mathrm{T}^{*}$ weighted magnitude images between M3 and M6 (Figure 2). Given the higher sensitivity of the phase image to subtle local paramagnetic shifts, this observation suggests the accrual over time of paramagnetic substances at the lesion edge. Whether this finding represents an ongoing attempt to enhance repair or the definitive proof of remyelination failure is uncertain.

At baseline, the strongest statistical predictors of a persistent phase rim are higher lesion volume and the presence of a $\mathrm{T} 2^{*}$ rim, 

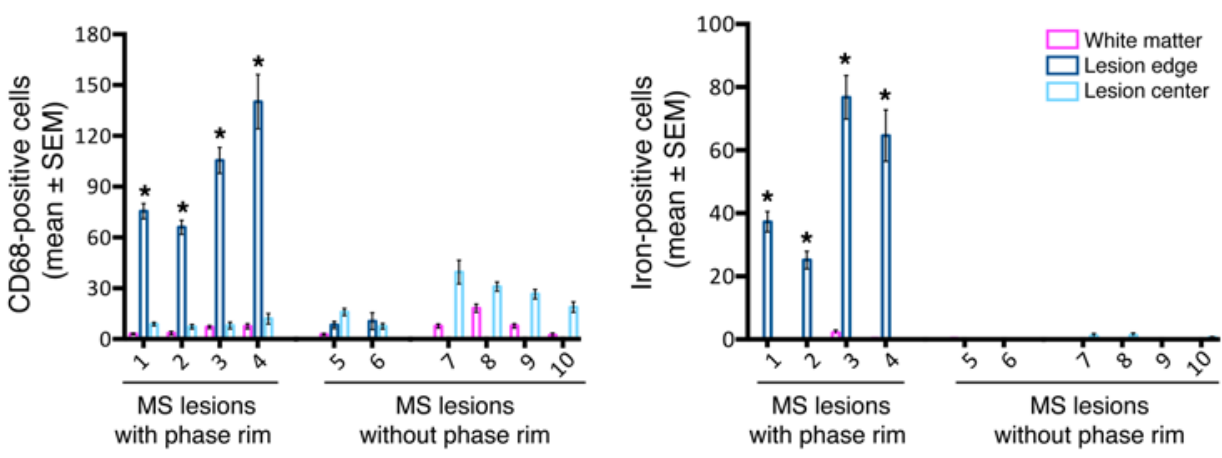

Figure 8. Distribution of CD68-positive and iron-positive cells in MS lesions with and without phase rim. Iron-laden CD68-positive macrophages/activated microglia were more common at the lesion edge than at the lesion center and within the surrounding white matter. On the other hand, lesions without phase rim showed varying degrees of non-iron-laden CD68-positive cells, not significantly different from the surrounding white matter. Lesions 1-4 belong to patient 1, lesions 5 and 6 to patient 2 , and lesions 7-10 to patient 3 (see Supplemental Appendix for details). ${ }^{*} P<0.0001$ in edge versus center and edge versus white matter (ANOVA, post hoc analysis and Bonferroni correction for multiple comparisons).

suggesting that lesions with this fate are more inflammatory even at onset. In this context, it is possible that the magnitude of the initial inflammation, rather than the intrinsic capacity of the injury response to contain lesion expansion, determines the lesion's fate. It is also likely that patient-specific factors, in particular age (as demonstrated here) $(48,49)$, but also potentially tissue energetics and vascular status, play important roles that should be further explored. Indeed, using an elegant parabiosis model, a recent study demonstrated that older mice have reduced ability to clean myelin debris and delayed capacity to shift the polarization of myeloid cells into repair-promoting phenotypes (30). Interestingly, in some leukocortical lesions, the rim became more prominent at the white/gray matter junction (Supplemental Figure 1). This phenomenon might be related to the presence of more iron in the subcortical $U$ fibers that may be released after demyelination, or perhaps to impaired macrophage migration into the gray matter (50) with consequent accumulation in the subcortical white matter.

Our preliminary observations suggest that short-term highdose corticosteroids - a common treatment for MS relapses - may limit the development of the persistent phase rim and the associated overall tissue damage. This effect might be mediated in part by the ability of corticosteroids to close the blood-brain barrier and thereby reduce the entrance of blood-derived inflammatory cells into the CNS; alternatively, corticosteroids might directly promote repair. This notion is in line with a previous report on improved recovery of magnetization transfer ratio values in steroid-treated enhancing lesions (51).

Finally, we performed an exploratory analysis of peripheral blood cytokines and chemokines (Supplemental Appendix). This analysis was not preplanned, and as a result we only had access to serum samples from a subset of the participants. Nonetheless, we found preliminary evidence that at the time of radiological relapse, participants with centripetal rather than centrifugal lesions harbor a prominent Th1-polarized cytokine profile (IL-12, IFN- $\gamma$, CXCL10 [IP10], IL-2, IL-15) that reduces during remission (Supplemental Figure 2).

Follow-up studies in larger populations could address several key questions: (a) Other than participant age, is the fate of newly forming lesions truly independent of clinical and demographic characteristics? (b) Do the 1-year trends in volume of lesions with and without phase rim continue over the longer term (up to 5 to 10 years) - which would suggest that this population of lesions might be the pathologically described "slowly expanding" or "smoldering" lesions or might the detrimental pathological processes underlying the persistent rim plateau or even reverse in some cases? (c) Are similar findings detectable at clinical magnetic field strength, which would facilitate broader, perhaps multicenter, application?

In summary, we suggest that the persistence of a lesion's phase rim is a negative prognostic factor in early lesion evolution, but also years later as shown by the pathological evaluation. Our results further suggest that therapeutic enhancement of tissue protection, as an adjunct to immunomodulatory therapies designed to reduce new lesion formation, should be a critical goal of treatment in early, inflammatory MS. In this context, successful prevention of the chronic phase rim might be useful as an outcome measure to gauge the success of such an approach.

\section{Methods}

\section{In vivo radiological assessment}

MRI acquisition. The 7T MRI protocol was as follows: (a) High-resolution (voxel size $0.2 \mathrm{~mm} \times 0.2 \mathrm{~mm} \times 1 \mathrm{~mm}$ ) $2 \mathrm{D}$ susceptibility-weighted gradient-echo sequence providing both $\mathrm{T} 2^{*}$-weighted magnitude contrast ("T2*") and susceptibility-weighted phase contrast ("phase") covered the lesion(s) of interest before contrast injection. (b) During injection of i.v. gadobutrol ( $0.1 \mathrm{mmol} / \mathrm{kg}$ over 1 minute), 10 repetitions of a 3D T1-weighted DCE sequence (32 seconds per repetition; voxel size $0.5 \mathrm{~mm} \times 0.5 \mathrm{~mm} \times 0.8 \mathrm{~mm}$ ) covered the lesion(s) of interest. This sequence was further repeated at variable time points over 20 minutes after contrast administration. (c) Whole-brain 3D T1-weighted magnetization-prepared rapid gradient echo (T1-MPRAGE; voxel size 0.7 $\mathrm{mm} \times 0.7 \mathrm{~mm} \times 0.7 \mathrm{~mm}$ ) was acquired before and after contrast injection (after $\sim 5$ and 16 minutes).

Additional MRI details have been previously described (16).

MRI analysis. An experienced neuroradiologist (D.S. Reich) read all the scans clinically, and a neurologist with experience in MS imaging (M. Absinta) analyzed the data. DCE and phase post-processing, as well as coregistration among images, were performed as previously described (16). 3D T1-MPRAGE images were coregistered and normalized using WhiteStripe (52), whereby intensity values are expressed in units of standard deviation (SD) of normal-appearing (extralesional) white matter. By convention, paramagnetic substances yield negative values in both phase and $2^{*}$ images $(12,16)$. Enhancing lesions were detected and classified as centrifugal (contrast enhancement first appears at the center of the lesion and over time spreads outward) and centripetal (contrast enhancement first appears at the lesion edge and then spreads inward) based on the DCE (21) or sequential T1-MPRAGE 


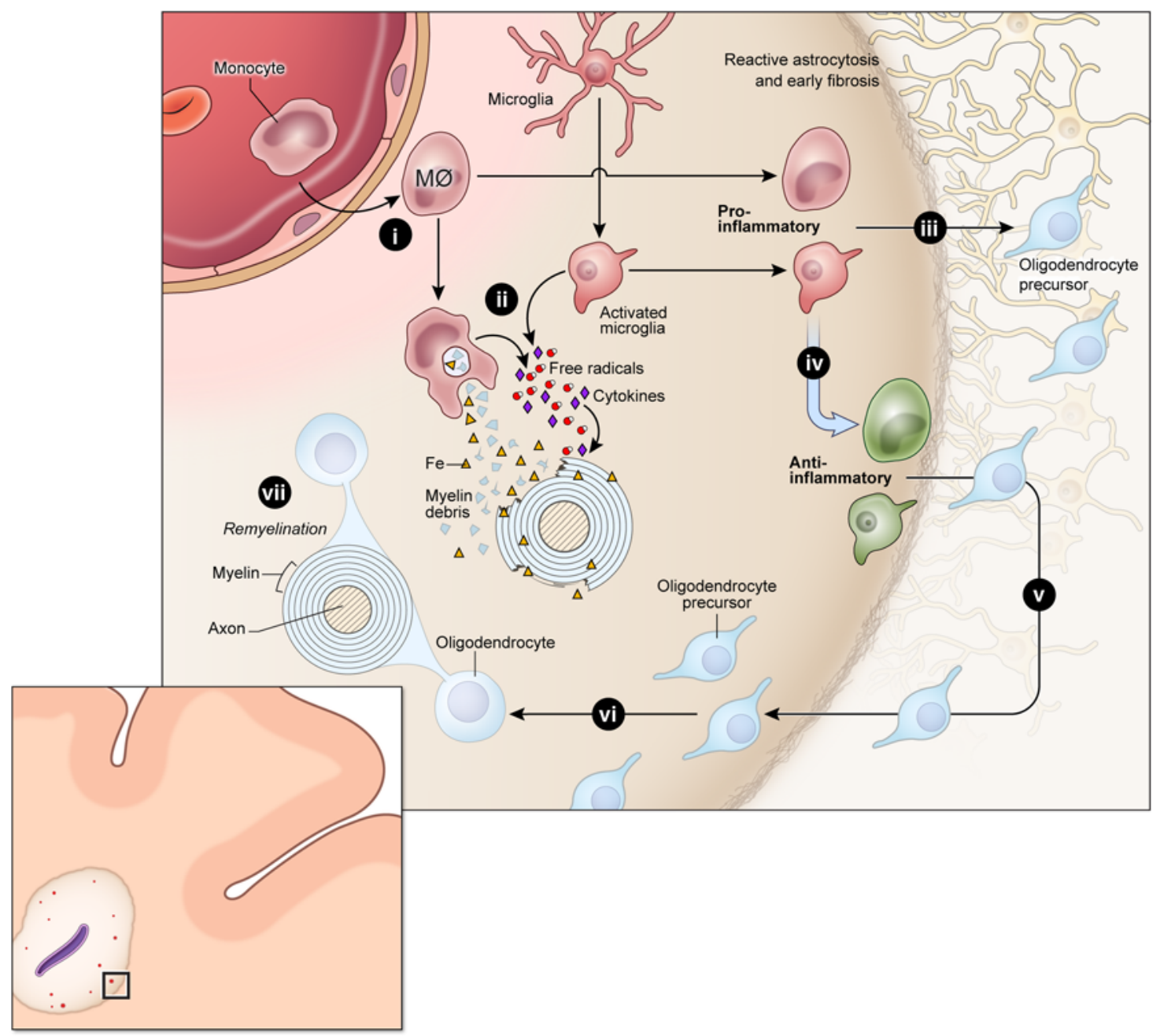

Figure 9. Phenomenological model of the origin and fate of the phase rim. In this hypothetical model, peripheral macrophages and activated microglia are recruited to the site of tissue damage (i). The potential roles of proinflammatory macrophages and microglia are shown in ii and iii: clearance of myelin debris, removal of free iron derived from the demyelinating process, production of free radicals, induction of the glial scar, and recruitment of oligodendrocyte precursors. The healing process is mediated by the shift from proinflammatory to antiinflammatory macrophages/microglia induced by interaction with the extracellular matrix and other factors (iv). Antiinflammatory macrophages/microglia help limit formation of a glial scar and promote migration of oligodendrocyte precursor cells into the demyelinated lesion (v), where they can mature into myelinating oligodendrocytes (vi) and remyelinate naked axons (vii). Any interruption of the healing process (e.g., failure of macrophages/microglia to acquire a fully antiinflammatory phenotype and subsequently clear macrophages/microglia) might trigger a vicious circle, resulting in persistence of the phase rim over at least the first year of lesion evolution.

images. Enhancing lesions with a maximum diameter $\leq 1.5 \mathrm{~mm}$, as well as confluent or re-enhancing lesions, were excluded from the analysis. In both centrifugal and centripetal lesions, for each time point, the presence or absence of a hypointense rim was assessed on $\mathrm{T} 2{ }^{*}$ and phase images. The rim thickness on phase images was also measured as previously described (16). The brain location of each lesion was recorded as periventricular, deep white matter, or cortical/juxtacortical. For each lesion, the lesion volume and intralesional mean T1 intensity on coregistered noncontrast 3D T1-MPRAGE images (paint tool, MIPAV software; NIH) were noted at the following time points: baseline/M1, M3, M6, and M12. Finally, the number and percentage of supratentorial discrete chronic lesions with phase rim on the baseline scan (50 axial 1-mm slices) were assessed for each participant.

Statistics. An analysis of covariance (ANCOVA) assessed the percentage change of lesion volume between M3 and M12, as (M3 M12)/M3, among lesion groups (centrifugal, centripetal with transient phase rim, and centripetal with persistent phase rim). Since a participant could have 1 or more lesions, a mixed-effects model evaluated the participant-specific contribution, but as this contribution was not statistically significant, it was excluded from the final model; thus, lesions from the same participant were treated as independent. The effect of rim persistence on worsening of T1 hypointensity ( $\geq 1$ unit of SD of normal-appearing white matter) between M3 and M12 was evaluated with Fisher's exact test. To test whether participants with lesions with persistent phase rim were older than those with transient rim, an ANOVA and unequal variance model (Levene's test for homogeneity of variance) were performed.

A logistic regression was used to establish the variables at baseline (lesion volume, presence or absence of a $\mathrm{T} 2^{*}$ rim, $\mathrm{T} 1$ hypointensity, participant age, brain lesion location) that predicted persistence of the phase rim at M12. Since the baseline lesion volume and T2* rim status were significant, the sensitivity and specificity of baseline 
lesion volume thresholds were estimated according to the presence or absence of a $\mathrm{T}^{*}$ rim at baseline. The effect of steroid administration at baseline on rim persistence was evaluated with Fisher's exact test. In all analyses, $P$ values of less than 0.05 were considered significant.

\section{Neuropathological evaluation}

Neuropathological evaluation of the formalin-fixed brain of a progressive MS patient (59 years old, 21 years of clinical disease duration) focused on 5 demyelinated MS lesions with phase rims that had been imaged at 7T MRI both in vivo (same imaging protocol as the in vivo cohort) and postmortem. An additional 6 demyelinated MS lesions without phase rim (on postmortem 7T MRI) from 2 other progressive MS patients were analyzed for comparison (details in Supplemental Appendix). MRI-matched histological sections were achieved via 7T MRI of the fixed brain and subsequent gross sectioning with an individualized, MRI-designed, 3D-printed cutting box (53). Formalin-fixed 5- or 10- $\mu \mathrm{m}$ cryosections or paraffin sections were stained with H\&E, Luxol fast blue/periodic acid-Schiff, Bielschowsky, and DAB-enhanced Turnbull staining (iron assessment). Immunohistochemical analysis for myelin proteolipid protein (PLP), aspartoacylase (ASPA, mature oligodendrocytes), OLIG2 (oligodendrocyte precursor cells), CD68 (macrophages/activated microglia), CD163 (mature tissue macrophages involved in resolution of inflammation), CD3 (T lymphocytes), CD8, CD4, CD20 (B lymphocytes), GFAP (reactive astrocytes), and SMI32 (axonal nonphosphorylated neurofilaments) was performed on representative slides (details in Supplemental Table 2). Selective double staining with ASPA/OLIG2 (mature oligodendrocytes/oligodendrocyte precursor cells), DAB-Turnbull/ CD68, DAB-Turnbull/GFAP, and CD163/CD68 was also performed. Bruce Trapp (Lerner Research Institute, Cleveland, Ohio, USA), provided a portion of the myelin PLP antibody, and Brian Popko (University of Chicago, Chicago, Illinois, USA), the ASPA antibody. For all antibodies, source and catalog number are provided in the Supplemental Appendix. Cell numbers were quantified with ImageJ software in 10 randomly taken fields (size $700 \mu \mathrm{m} \times 525 \mu \mathrm{m}$ ), using $\times 20$ magnification, at different locations (lesion center, lesion edge, and white matter surrounding the lesion) and statistically analyzed using
ANOVA analysis and post-hoc comparisons (Bonferroni-corrected for multiple comparisons; $P<0.05)$.

\section{Study approval}

Patients were recruited under an IRB-approved natural history protocol (NINDS, NIH). All patients provided informed consent prior to their participation in the study. The 3 formalin-fixed brains were attained at autopsy after consent was obtained from the next of kin.

\section{Author contributions}

MA and DSR contributed to study concept and design. MA, PS, MS, ECL, JO, ICMC, and DSR contributed to acquisition of data. MA, PS, ECL, JO, TW, AM, and DSR contributed to data analysis. MA, PS, MF, SJ, ICMC, and DSR contributed to interpretation and critical revision of the manuscript for important intellectual content. DSR supervised the study.

\section{Acknowledgments}

The Intramural Research Program of NINDS supported this study. M. Absinta was also partially supported by a National Multiple Sclerosis Society postdoctoral fellowship, award FG 2093-A-1. We thank the study participants, the Neuroimmunology Clinic for recruiting and evaluating the patients and for coordinating the scans, and the NIH Functional Magnetic Resonance Imaging Facility. We thank Govind Nair for valuable advice and assistance with $3 \mathrm{~T}$ data acquisition. We thank Seung-Kwon Ha for advice and assistance with histological assessment. We also thank Luisa Vuolo and Xiaozhen Li for helping with the 7T data acquisition, Blake Dewey for helping with the data analysis pipeline, Alan Hoofring for his artistic contribution, Bruce Trapp for providing the myelin proteolipid protein antibody, and Brian Popko for providing the aspartoacylase antibody.

Address correspondence to: Daniel S. Reich, Translational Neuroradiology Unit/NINDS/NIH, 10 Center Drive MSC 1400, Building 10 Room 5C103, Bethesda, Maryland 20852, USA. Phone: 301.496.1801; E-mail: reichds@ninds.nih.gov.
1. Lucchinetti C, Bruck W, Parisi J, Scheithauer B, Rodriguez M, Lassmann H. Heterogeneity of multiple sclerosis lesions: implications for the pathogenesis of demyelination. Ann Neurol. 2000;47(6):707-717.

2. Patrikios $P$, et al. Remyelination is extensive in a subset of multiple sclerosis patients. Brain. 2006;129(pt 12):3165-3172.

3. Kuhlmann T, Miron V, Cui Q, Wegner C, Antel J, Bruck W. Differentiation block of oligodendroglial progenitor cells as a cause for remyelination failure in chronic multiple sclerosis. Brain. 2008;131(pt 7):1749-1758.

4. Chang A, Tourtellotte WW, Rudick R, Trapp BD. Premyelinating oligodendrocytes in chronic lesions of multiple sclerosis. $\mathrm{N} \mathrm{Engl} \mathrm{J} \mathrm{Med.}$ 2002;346(3):165-173.

5. Kotter MR, Stadelmann C, Hartung HP. Enhancing remyelination in disease - can we wrap it up? Brain. 2011;134(pt 7):1882-1900.

6. Kremer D, Aktas O, Hartung HP, Kury P. The complex world of oligodendroglial differentia- tion inhibitors. Ann Neurol. 2011;69(4):602-618.

7. Shechter R, Schwartz M. CNS sterile injury: just another wound healing? Trends Mol Med. 2013;19(3):135-143.

8. Franklin RJ, Gallo V. The translational biology of remyelination: Past, present, and future. Glia. 2014;62(11):1905-1915.

9. Frischer JM, et al. Clinical and pathological insights into the dynamic nature of the white matter multiple sclerosis plaque. Ann Neurol. 2015;78(5):710-721.

10. Bramow S, et al. Demyelination versus remyelination in progressive multiple sclerosis. Brain. 2010;133(10):2983-2998.

11. van der Valk P, De Groot CJ. Staging of multiple sclerosis (MS) lesions: pathology of the time frame of MS. Neuropathol Appl Neurobiol. 2000;26(1):2-10.

12. Hammond KE, et al. Quantitative in vivo magnetic resonance imaging of multiple sclerosis at 7 Tesla with sensitivity to iron. Ann Neurol. 2008;64(6):707-713.
13. Pitt D, et al. Imaging cortical lesions in multiple sclerosis with ultra-high-field magnetic resonance imaging. Arch Neurol. 2010;67(7):812-818.

14. Yao B, et al. Chronic multiple sclerosis lesions: characterization with high-field-strength MR imaging. Radiology. 2012;262(1):206-215.

15. Hagemeier J, et al. Iron deposition in multiple sclerosis lesions measured by susceptibilityweighted imaging filtered phase: a case control study. J Magn Reson Imaging. 2012;36(1):73-83.

16. Absinta M, et al. Seven-tesla phase imaging of acute multiple sclerosis lesions: a new window into the inflammatory process. Ann Neurol. 2013;74(5):669-678.

17. Dal-Bianco A, et al. Veins in plaques of multiple sclerosis patients - a longitudinal magnetic resonance imaging study at 7 Tesla. Eur Radiol. 2015;25(10):2913-2920.

18. Bian $\mathrm{W}$, et al. A serial in vivo 7T magnetic resonance phase imaging study of white matter lesions in multiple sclerosis. Mult Scler. 2013;19(1):69-75. 
19. Bagnato F, et al. Tracking iron in multiple sclerosis: a combined imaging and histopathological study at 7 Tesla. Brain. 2011;134(pt 12):3602-3615.

20. Walsh AJ, et al. Multiple sclerosis: validation of MR imaging for quantification and detection of iron. Radiology. 2013;267(2):531-542.

21. Gaitan MI, et al. Evolution of the blood-brain barrier in newly forming multiple sclerosis lesions. Ann Neurol. 2011;70(1):22-29.

22. Gaitan MI, Sati P, Inati SJ, Reich DS. Initial investigation of the blood-brain barrier in MS lesions at 7 tesla. Mult Scler. 2013;19(8):1068-1073.

23. Kurtzke JF. Rating neurologic impairment in multiple sclerosis: an expanded disability status scale (EDSS). Neurology. 1983;33(11):1444-1452.

24. Trapp BD, Peterson J, Ransohoff RM, Rudick $\mathrm{R}$, Mork S, Bo L. Axonal transection in the lesions of multiple sclerosis. $\mathrm{N} \mathrm{Engl} \mathrm{J} \mathrm{Med.}$ 1998;338(5):278-285.

25. Mehta V, et al. Iron is a sensitive biomarker for inflammation in multiple sclerosis lesions. PLoS One. 2013;8(3):e57573.

26. Vogel DY, et al. Macrophages in inflammatory multiple sclerosis lesions have an intermediate activation status. J Neuroinflammation. 2013;10:35.

27. Murray PJ, et al. Macrophage activation and polarization: nomenclature and experimental guidelines. Immunity. 2014;41(1):14-20.

28. Raposo C, Schwartz M. Glial scar and immune cell involvement in tissue remodeling and repair following acute CNS injuries. Glia. 2014;62(11):1895-1904

29. Peferoen LA, et al. Activation status of human microglia is dependent on lesion formation stage and remyelination in multiple sclerosis. J Neuropathol Exp Neurol. 2015;74(1):48-63.

30. Miron VE, et al. M2 microglia and macrophages drive oligodendrocyte differentiation during CNS remyelination. Nat Neurosci. 2013;16(9):1211-1218.

31. Bogie JF, Stinissen P, Hendriks JJ. Macrophage subsets and microglia in multiple sclerosis. Acta Neuropathol. 2014;128(2):191-213.

32. Cherry JD, Olschowka JA, O'Banion MK. Neuroinflammation and M2 microglia: the good, the bad, and the inflamed. J Neuroinflammation. 2014;11:98.

33. Chen JT, Collins DL, Atkins HL, Freedman MS, Arnold DL, Canadian MS/BMT Study Group. Magnetization transfer ratio evolution with demyelination and remyelination in multiple sclerosis lesions. Ann Neurol. 2008;63(2):254-262.

34. Reich DS, et al. Sample-size calculations for short-term proof-of-concept studies of tissue protection and repair in multiple sclerosis lesions via conventional clinical imaging. Mult Scler. 2015;21(13):1693-1704.

35. Bruck W, Bitsch A, Kolenda H, Bruck Y, Stiefel M, Lassmann H. Inflammatory central nervous system demyelination: correlation of magnetic resonance imaging findings with lesion pathology. Ann Neurol. 1997;42(5):783-793.

36. van Walderveen MA, et al. Histopathologic correlate of hypointense lesions on T1-weighted spin-echo MRI in multiple sclerosis. Neurology. 1998;50(5):1282-1288.

37. van Waesberghe JH, et al. Axonal loss in multiple sclerosis lesions: magnetic resonance imaging insights into substrates of disability. Ann Neurol. 1999;46(5):747-754.

38. Bitsch A, Kuhlmann T, Stadelmann C, Lassmann H, Lucchinetti C, Bruck W. A longitudinal MRI study of histopathologically defined hypointense multiple sclerosis lesions. Ann Neurol. 2001;49(6):793-796.

39. Wisnieff C, Ramanan S, Olesik J, Gauthier S, Wang Y, Pitt D. Quantitative susceptibility mapping (QSM) of white matter multiple sclerosis lesions: Interpreting positive susceptibility and the presence of iron. Magn Reson Med. 2015;74(2):564-570.

40. He X, Yablonskiy DA. Biophysical mechanisms of phase contrast in gradient echo MRI. Proc Natl Acad Sci U S A. 2009;106(32):13558-13563.

41. Li W, Wu B, Avram AV, Liu C. Magnetic susceptibility anisotropy of human brain in vivo and its molecular underpinnings. Neuroimage. 2012;59(3):2088-2097.

42. Yablonskiy DA, Luo J, Sukstanskii AL, Iyer A, Cross AH. Biophysical mechanisms of MRI signal frequency contrast in multiple sclerosis. Proc Natl
Acad Sci U S A. 2012;109(35):14212-14217.

43. Hametner S, Wimmer I, Haider L, Pfeifenbring S, Bruck W, Lassmann H. Iron and neurodegeneration in the multiple sclerosis brain. Ann Neurol. 2013;74(6):848-861.

44. Stephenson E, Nathoo N, Mahjoub Y, Dunn JF, Yong VW. Iron in multiple sclerosis: roles in neurodegeneration and repair. Nat Rev Neurol. 2014;10(8):459-468.

45. Todorich B, Pasquini JM, Garcia CI, Paez PM, Connor JR. Oligodendrocytes and myelination: the role of iron. Glia. 2009;57(5):467-478.

46. Schonberg DL, McTigue DM. Iron is essential for oligodendrocyte genesis following intraspinal macrophage activation. Exp Neurol. 2009;218(1):64-74.

47. Schonberg DL, Goldstein EZ, Sahinkaya FR, Wei P, Popovich PG, McTigue DM. Ferritin stimulates oligodendrocyte genesis in the adult spinal cord and can be transferred from macrophages to NG2 cells in vivo. J Neurosci. 2012;32(16):5374-5384.

48. Goldschmidt T, Antel J, Konig FB, Bruck W, Kuhlmann T. Remyelination capacity of the MS brain decreases with disease chronicity. Neurology. 2009;72(22):1914-1921.

49. Rawji KS, Mishra MK, Michaels NJ, Rivest S, Stys PK, Yong VW. Immunosenescence of microglia and macrophages: impact on the ageing central nervous system. Brain. 2016;139(pt 3):653-661.

50. Prins M, et al. Discrepancy in CCL2 and CCR2 expression in white versus grey matter hippocampal lesions of Multiple Sclerosis patients. Acta Neuropathol Commun. 2014;2:98.

51. Richert ND, Ostuni JL, Bash CN, Leist TP, McFarland HF, Frank JA. Interferon beta-1b and intravenous methylprednisolone promote lesion recovery in multiple sclerosis. Mult Scler. 2001;7(1):49-58.

52. Shinohara RT, et al. Statistical normalization techniques for magnetic resonance imaging. Neuroimage Clin. 2014;6:9-19.

53. Absinta M, et al. Postmortem magnetic resonance imaging to guide the pathologic cut: individualized, 3-dimensionally printed cutting boxes for fixed brains. J Neuropathol Exp Neurol. 2014;73(8):780-778. 